\title{
Enantioselective $\alpha, \delta$-Difunctionalization of Dienes Initiated by Rh-Catalyzed Conjugate Addition
}

Christopher J. C. Cooze, Wesley McNutt, Markus D. Schoetz, Bohdan Sosunovych, Svetlana Grigoryan, Rylan J. Lundgren

Department of Chemistry, University of Alberta, Edmonton, Alberta, T6G 2G2, Canada; rylan.lundgren@alberta.ca

\begin{abstract}
Metal-catalyzed enantioselective conjugate additions are highly reliable methods for stereoselective synthesis, however multi-component reactions that are initiated by conjugate arylation of acyclic $\pi$-systems are rare. These processes generally proceed with poor diastereoselectivity while requiring basic, moisture sensitive organometallic nucleophiles. Here we show that Rh-catalysts supported by a tetrafluorobenzobarrelene ligand (Ph-tfb) enable the enantio-, diastereo-, and Z-selective $\alpha, \delta$-difunctionalization of electron-deficient 1,3-dienes with organoboronic acid nucleophiles and aldehyde electrophiles to generate Z-homoallylic alcohols with three stereocenters. The reaction accommodates diene substrates activated by ester, amide, ketone, or aromatic groups and can be used to couple aryl, alkenyl, or alkyl aldehydes. Diastereoselective functionalization of the Z-olefin unit in the addition products allow for the generation of compounds with five stereocenters in high $d r$ and ee. Mechanistic studies suggest aldehyde allylrhodation is the rate determining step, and unlike reactions of analogous Rh-enolates, the Rh-allyl species generated by $\delta$-arylation undergoes aldehyde trapping rather than protonolysis, even when water is present as a co-solvent. These findings should have broader implications in the use of privileged metal-catalyzed conjugate addition reactions as entry points towards the preparation of acyclic molecules containing non-adjacent stereocenters.
\end{abstract}




\section{INTRODUCTION}

Transition-metal catalyzed enantioselective conjugate addition reactions are among the most well-studied and reliable methods for the stereocontrolled formation of carbon-carbon bonds. ${ }^{1}$ They have enjoyed widespread use in natural products synthesis, medicinal chemistry campaigns, and the large scale preparation of enantioenriched small molecules (Fig 1a). ${ }^{2}$ The Rh-catalyzed conjugate arylation of electron-deficient alkenes using boronic acid derived nucleophiles arguably provides the most accommodating platform to generate $\beta$-arylated stereocenters, achieving high selectivity profiles across structurally diverse classes of substrates under weakly basic conditions. ${ }^{1 \mathrm{~b}, 1 \mathrm{~d}}$ While studied less $^{2}$ intensely than additions to alkenes, electron-poor dienes can undergo metal-catalyzed conjugate addition to generate a new stereocenter $\delta$ to an activating group (Fig $1 \mathrm{~b}$ ). ${ }^{3}$ The olefin unit in the product is primed for subsequent functionalization, making this an ideal entry point into the preparation of acyclic molecules with multiple stereocenters. Achieving positional selectivity for nucleophile addition ( $\beta$ vs $\delta$ ) while forming products with high olefin regio- and stereoselectivity ( $E$ vs $Z, \alpha, \beta$ - vs $\beta, \gamma$-unsaturation) with acyclic diene substrates remains a challenge. Examples are restricted to Cu-catalyzed alkylations ${ }^{4}$ and allylations, ${ }^{5}$ and Co-catalyzed alkynylations, ${ }^{6}$ these processes give E-alkene products. The Z- and enantioselective $\delta$-arylation of carbonyl-activated dienes with aryl boroxines can be achieve with Irbased catalysts, although products of the reactions are typically isolated after isomerization to the $\alpha, \beta$ unsaturated species or hydrogenation of the alkene. ${ }^{7}$ Given the current difficulty in preparing acyclic molecules with multiple stereocenters in a single step, ${ }^{8}$ the development of new processes that leverage the mechanistic steps of metal-catalyzed conjugate additions in multi-component couplings would be valuable. 


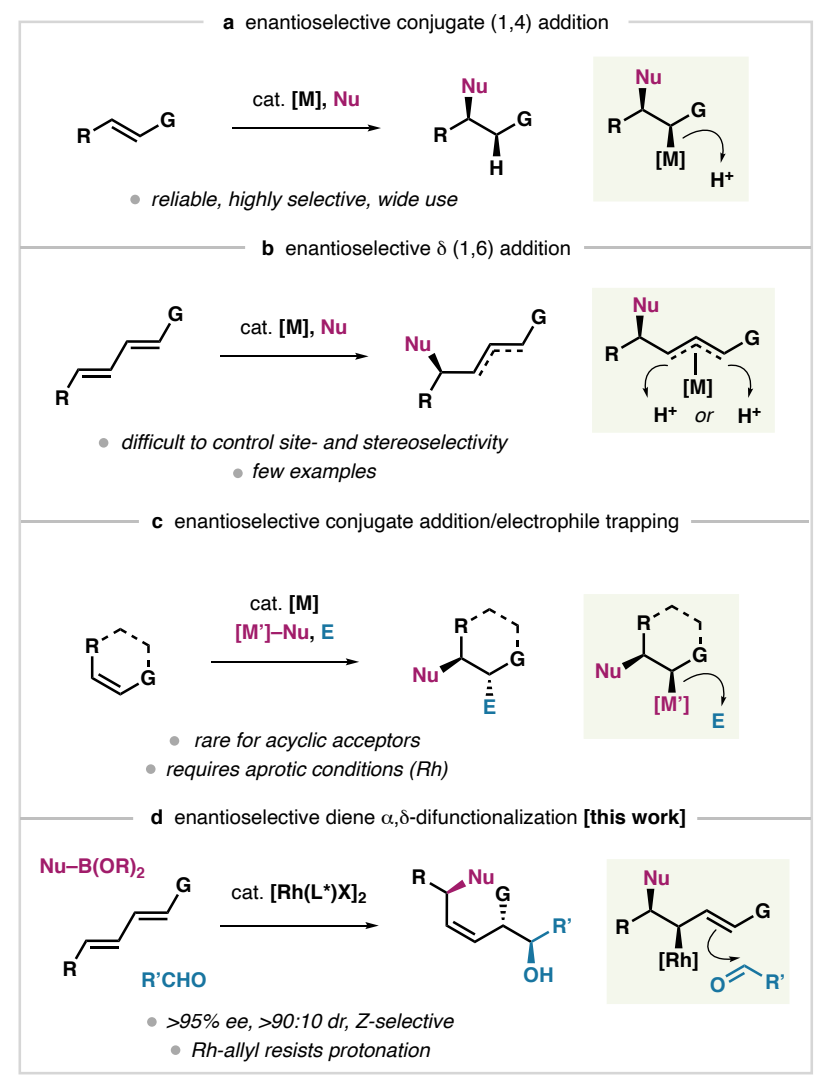

Figure 1. Overview of methods for the metal-catalyzed, enantioselective addition of carbon nucleophiles to electron-poor dienes ( $G$ = electron-withdrawing group).

Metal-catalyzed conjugate addition reactions generate nucleophilic intermediates after the initial addition step (Fig 1). These species' are usually protonated to generate hydroarylation products. In contrast, in a limited number of reports have shown that the nucleophilic metal intermediate generated in an enantioselective conjugate addition reaction, typically an enolate, can be intercepted by non-proton electrophiles generating products with two adjacent stereocenters (Fig 1c). ${ }^{1 \mathrm{c}, 1 \mathrm{~d}, 9}$ This approach is generally restricted to cyclic conjugate acceptors like cycloenones or to intramolecular reactions with tethered electrophiles. ${ }^{1 c, 1 d, 10}$ In the case of Rh-catalyzed $\alpha, \beta$-difunctionalizations, the use of Ti-aryl ${ }^{11}$ or 9-BBN-aryl ${ }^{12}$ reagents under non-protic conditions instead of aryl boronic acids is required to suppress rapid protonation of enolate intermediates. ${ }^{13}$ By analogy, intercepting the nucleophilic metal-allyl intermediated generated by $\delta$-addition to a 1,3-diene with an external electrophile, like an aldehyde, would allow the preparation of acyclic products with 1,4-stereocenters (Fig 1d). ${ }^{8 b}$ While several 
enantioselective diene carbo-difunctionalization reactions are known, none functionalize acyclic substrates to generate non-adjacent stereocenters at the $\alpha$ - and $\delta$-positions. ${ }^{14}$ We recently developed the racemic Z-selective reductive coupling of electron-deficient dienes and aldehydes ${ }^{15}$ and questioned whether the Rh-allyl intermediate generated in an enantioselective $\delta$-arylation could be trapped in a similar way. ${ }^{16}$ Achieving the stereoselective diene $\alpha, \delta$-difunctionalization triggered by conjugate arylation would require high selectivity at several mechanistic steps (Fig 2) including: (i) suppression of direct aldehyde arylation from Rh-aryl intermediate $\mathbf{A},{ }^{17}$ (ii) site and enantioselective diene $\delta$-arylation, ${ }^{18}$ (iii) aldehyde trapping of Rh-allyl intermediate $\mathbf{B}$ without $\eta^{1}-\eta^{3}-\eta^{1}$ allyl face-swapping or chain-walking isomerization, ${ }^{19}$ and (iv) selective protonolysis of only Rh-alkoxide intermediate $\mathbf{C}$ and not $\mathbf{B}$ which would lead to hydroarylation products. Here, we show that Rh-catalysts with chiral tetrafluorobenzobarrelene ligands enable the enantioselective $\delta$-arylation of organoboronic acid nucleophiles to dienes followed by the Z-syn selective $\alpha$-trapping of aldehydes. (Fig 1d). The three-component reaction products can be readily converted in linear compounds with five adjacent stereocenters. Mechanistic studies show that Rh-allyl intermediates generated by the $\delta$-arylation of dienes are uniquely suited to undergo stereoselective interception with non-proton electrophiles, contrasting the reactivity of related Rhenolate intermediates. 


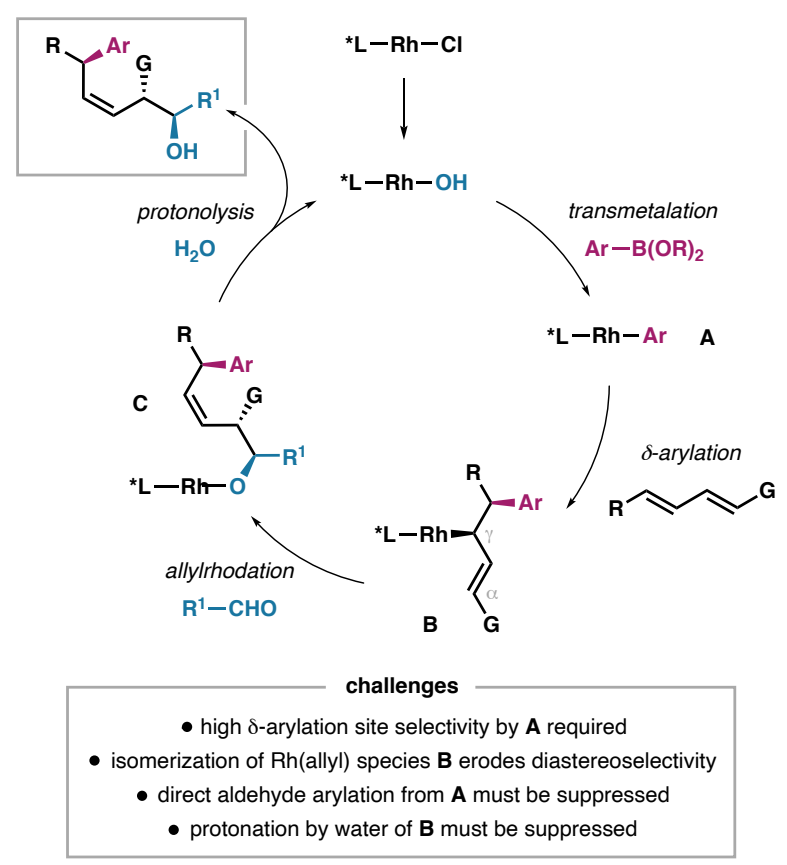

Figure 2. Mechanistic framework for the Rh-catalyzed enantioselective $\alpha, \delta$-difunctionalization of dienes.

\section{RESULTS AND DISCUSSION}

\section{Reaction Development}

With the aim of developing a three-component coupling reaction initiated through a metalcatalyzed $\delta$-arylation, a variety of catalysts and reaction conditions were surveyed to find that the enantioselective $\alpha, \delta$-difunctionalization of diene 1 with 3-bromophenylboronic acid and 3chlorobenzaldehyde could be achieved to give 2 (Fig 3). Using a Rh-complex supported by Nishimura's chiral tetrafluorobenzobarrelene ligand (Ph-tfb), ${ }^{7,20} 2$ was generated in $98 \%$ yield, $98 \%$ ee and $97: 3 d r$ (2:sum of others) with high Z-selectivity (>98:2). Other chiral diene ligands commonly used in enantioselective conjugate additions proved inferior to $\mathrm{Ph}$-tfb, including the structurally related $\mathrm{Ph}$-bod ligand $(98 \%$ ee vs $21 \%$ ee, Fig 3a). Either arylboronic acids or pinacol esters could be used as the arylating reagent (Fig 3b). A 10:1 mixture of DMF/water was the optimal solvent, although DMF could be replaced with ethereal solvents like dioxane at the expense of reaction rate. The presence of water was essential, no product is observed without it. Reactions conducted at decreased concentration and at room temperature led to a reduced diastereoselectivity (from 97:3 to $90: 10$ ), likely because the Rhallyl intermediate is long-lived enough under these conditions to undergo $\eta^{1}-\eta^{3}-\eta^{1}$ isomerization with 
allyl face-swapping (see below for a discussion). The catalyst loadings could be reduced to 1 mol\% total $\mathrm{Rh} /$ ligand with similar yields and selectivities. The use of $\operatorname{Ir}(\mathrm{tfb})$-based catalysts resulted in no product formation ( $<2 \%$ yield, see the SI for details).

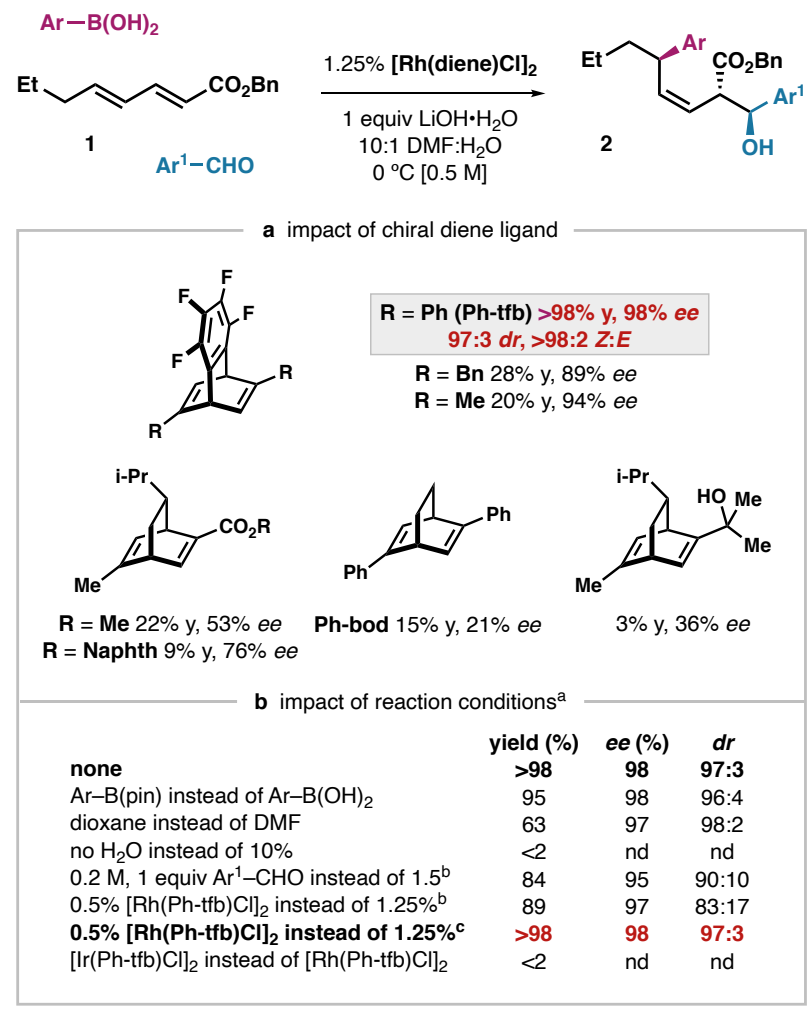

Figure 3. Impact of chiral ligands and reaction conditions on the enantioselective diene $\alpha, \delta$ difunctionalization; 1:aldehyde: $\mathrm{ArB}(\mathrm{OR})_{2}=1: 1.5: 2$, yields determined by ${ }^{1} \mathrm{H} N \mathrm{NMR}$, ee determined by chiral HPLC, $d r$ is the ratio of product to all other isomers, see the SI for details. 'Unless noted using $[\mathrm{Rh}(\mathrm{Ph}-\mathrm{tfb}) \mathrm{Cl}]_{2} .{ }^{\mathrm{b}}$ At room temperature. ${ }^{\mathrm{c}} 9$ hour reaction $\mathrm{Ar}=3-\mathrm{BrC}_{6} \mathrm{H}_{4}, \mathrm{Ar}{ }^{\prime}=3-\mathrm{ClC}_{6} \mathrm{H}_{4}$.

\section{Reaction Scope and Application}

The Rh-catalyzed diene $\alpha, \delta$-difunctionalization process enables access to chiral Z-homoallylic alcohols with three stereocenters (Fig 4). For both electron-rich (3-5) and electron-poor $(\mathbf{6}, \mathbf{7}, \mathbf{1 0})$ aryl boron nucleophiles, the products diastereo- and enantioselectivities remain high ( $\geq 90: 10 d r, \geq 90 \%$ ee, $>50 \%$ yield). Potentially reactive units like aryl halides $(2,8,11), \mathrm{NH}$-groups $(4,5)$, nitriles $(7)$, aryl silanes (11), and aryl acrylates (12) are tolerated on the aryl boron group. The aryl aldehyde partner can feature electron-donating groups (15) or electron-withdrawing groups $(\mathbf{1 6}, 17)$ as well as phenol $(\mathbf{1 8})$ or ortho- 
Br substitution (19). The dienoate partner can feature either alkyl or aryl groups at the $\delta$-position $(2,20-$ 23). Variation of the diene's ester substituent had little impact on selectivity (Me, Bn, long-chain alkyl, each $\geq 95 \%$ ee) with uniformly high yields and selectivities observed. Dienamides, including those featuring the Weinreb amide engaged in the reaction to give products as nearly single diastereomers and in $\geq 95 \%$ ee $(\mathbf{2 5}, \mathbf{2 6})$. Dienyl arenes could be used in place of carbonyl-activated dienes to prepare allylic substrates with syn-1,2-diaryl units in $>95 \%$ ee (27-29). A dienone substrate underwent $\alpha, \delta$ difunctionalization with reduced diastereoselectivity (70:30) to give $\mathbf{3 0}$ but with high enantioselectivity for both products ( $97 \%$ ee major, $95 \%$ ee minor). Less successful substrates include those with aryl iodide groups (13), pyridyl aldehydes, and $\delta$-disubstituted dienoates (see the SI for more details).

The enantioselective diene addition process can be used to access other classes of products, including those featuring skipped 1,4-(E,Z)-dienes by the use of alkenyl boronic ester nucleophiles (31), 1,5-dienes by the use of $\alpha, \beta$-unsaturated aldehyde partners (32) and dialkyl Z-homoallylic alcohols by the use of alkyl aldehydes (33). When a $\delta$-unsubstituted aryl diene is used as a reaction partner $\left(\mathrm{R}^{1}=\right.$ $H$ ), the sense of enantio-addition arising from the $\delta$-arylation step is relayed to the aldehyde allyIrhodation step to give products with two stereocenters in $94 \%$ ee, remote from the initial arylation (34). ${ }^{14 c}$ Collectively these scope studies demonstrate that under suitable conditions, enantioselective Rh-catalyzed conjugate addition can be relayed to electrophile trapping by Rh-allyl intermediates with high fidelity to generate stereochemically rich, acyclic molecules. 

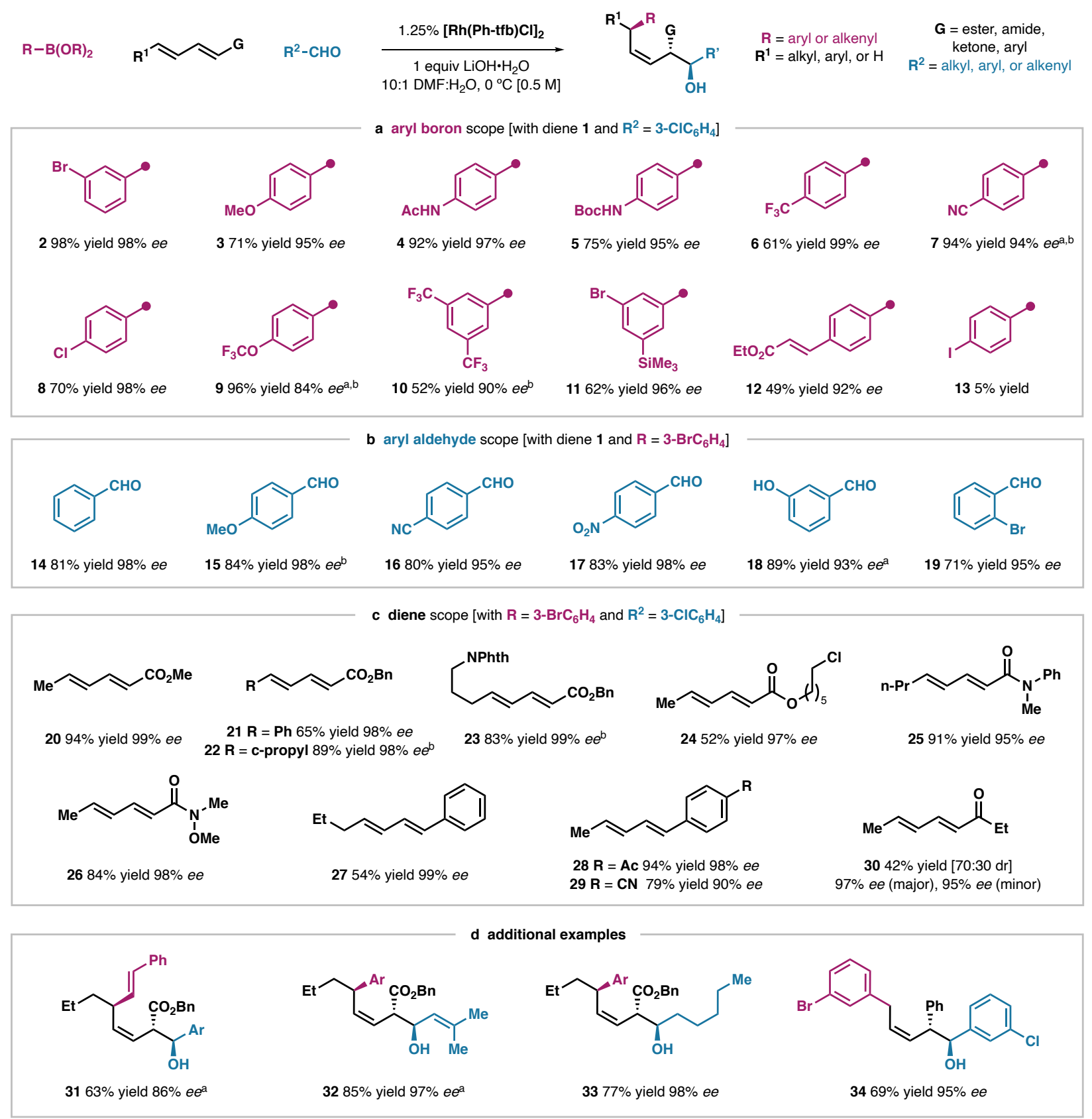

Figure 4. Scope of the Rh-catalyzed, enantioselective diene $\alpha, \delta$-difunctionalization dienes. Unless noted, yields are of isolated material with $\geq 95: 5 d r$. ${ }^{a}$ Yield determined by ${ }^{1} \mathrm{H} N M R$. ${ }^{b} d r$ of isolated material 94:6-90:10. See SI for full details.

The products of the $\alpha, \delta$-difunctionalization feature three stereocenters separated by a Z-olefin unit which can be converted into other acyclic molecules containing a diversity of functional groups with high diastereoselectivity (Fig 5). First, 35 was prepared on the gram scale $(1.3 \mathrm{~g}, 87 \%$ yield, $99 \%$ ee using $0.5 \mathrm{~mol} \%$ catalyst). This species could be quantitatively converted by catalytic hydrogenation to 
36 which contains remote 1,4-stereocenters. Dihydroxylation of 35 followed by $\mathrm{LiAlH}_{4}$ reduction of the intermediate lactone afforded tetrahydroxy product 37 as a single stereoisomer in $39 \%$ overall yield. Diastereoselective alkene epoxidation using $\mathrm{V}(\mathrm{O})(\mathrm{acac})_{2}$ and $t$ - $\mathrm{BuOOH}$ or dibromination with $\left[\mathrm{N}\left(\mathrm{Me}{ }_{3} \mathrm{Bn}\right)\right]$ $\mathrm{Br}_{3}$ yielded acyclic products with five consecutive tertiary stereocenters $(38,39)$. The syn- $\beta$-hydroxy ester unit is also poised for further functionalization, for example in the synthesis of amino alcohol $\mathbf{4 0 ,}$ generated in $50 \%$ overall yield and $99 \%$ ee by ester hydrolysis of $\mathbf{3 6}$ followed by Curtius rearrangement and carbamate ring opening.

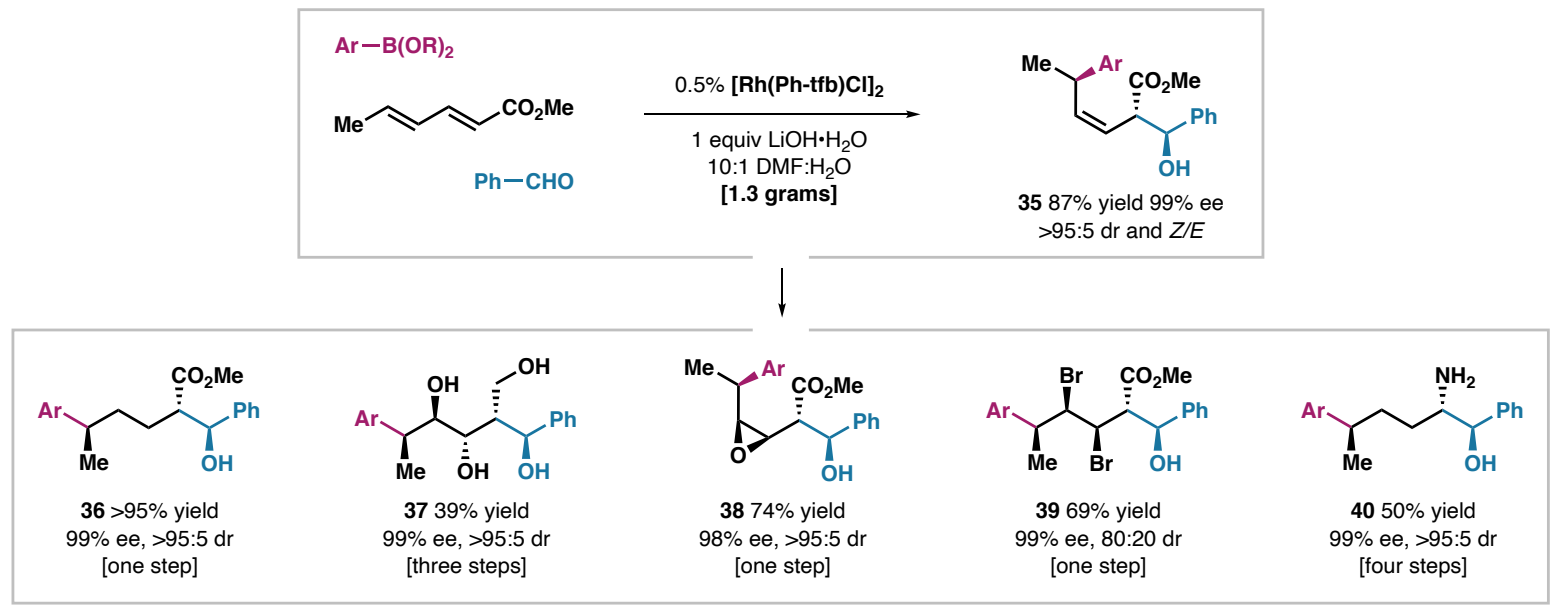

Figure 5. Functionalization of $\alpha, \delta$-difunctionalization product 35 to generate stereochemically rich acyclic compounds. $\mathrm{Ar}=4-\mathrm{OMeC}_{6} \mathrm{H}_{4}$. See the $\mathrm{SI}$ for details and reaction conditions.

\section{Mechanistic Analysis}

Mechanistic analysis was conducted to better understand the origin of high chemo- and stereoselectivity in the Rh-catalyzed diene $\alpha, \delta$-difunctionalization reaction and to identify how the nature of each reaction component impacts the process. The stereochemistry of the products agree with the established sense of metal/(Ph-tfb)-catalyzed conjugate addition to electron-poor dienes ${ }^{7 a}$ and $\mathrm{Rh}(\mathrm{cod})$ catalyzed reductive aldehyde allylation. ${ }^{15,19 a}$ Coordination to minimize steric clashing between the Rgroup of the diene substrate and the aryl ligand on Rh as well as position the electron-withdrawing group (G) in the open pocket created by the chiral diene ligand sets the stereochemistry at the $\delta$-position (Fig $6 a$, the sense of addition is the reverse for $\beta$-arylations, see the SI for additional details). The Z-syn 
selectivity is consistent with a six-membered transition state involving a Rh-allyl nucleophile and the incoming aldehyde (Fig 6b). ${ }^{21}$ The minor diastereomer generated in the reaction, formed in $\sim 2$ to $10 \%$ depending on the combination of substrates, is the Z-syn addition product arising from ally face swapping of intermediate B to Rh-allyl B' (Figure 6b). Formation of the minor diastereomer increases when using less effective catalysts or when the initial aldehyde concentration is lowered, suggesting if trapping of $\mathbf{B}$ is too slow, allyl isomerization occurs. Moderate to high Z-syn diastereoselectivity is observed with achiral catalyst like $[\mathrm{Rh}(\operatorname{cod}) \mathrm{Cl}]_{2}$, suggesting that the chiral ligands do not impact the selectivity at this step.

The reaction demonstrates a brief induction period which arises from the initial conversion of the $[(\mathrm{Ph}-\mathrm{tfb}) \mathrm{RhCl}]_{2}$ pre-catalysts to the active (Ph-tfb)RhOH catalyst. ${ }^{1 \mathrm{~d}}$ When using $[(\mathrm{Ph}-\mathrm{tfb}) \mathrm{RhOH}]_{2}, \mathrm{LiOH}$ is not required but its presence increases reaction rates. Preliminary kinetic data obtained by variable time normalization plots ${ }^{22}$ using $[(\mathrm{Ph}-\mathrm{tfb}) \mathrm{RhOH}]_{2}$ as the catalyst show the reaction to be first order in [Rh] and aldehyde, approximately zero order in diene, and partial positive order in aryl boronic acid (see the SI for details). These observations suggest that aldehyde allylation from B is the rate determining step.

The (Ph-tfb)Rh-allyl intermediate is uniquely selective for aldehyde trapping over protonation despite the high concentration of water present in the reaction $(5 \mathrm{M})$. To probe this behaviour, a series of competition and rate experiments were conducted. In the absence of aldehyde, diene 1 undergoes addition and protonation to generate $\delta$-hydroarylation product 41 in $90 \%$ yield and $95 \%$ ee (Fig 6c). The hydroarylation reaction is $\sim 20$ times slower than $\alpha, \delta$-difunctionalization with aldehyde electrophiles. Given the similar mechanistic pathway, Rh-allyl protonolysis is likely also the slow step in direct $\delta$ arylations of dienes (Fig 6c). Contrasting the reactivity of dienes, simple $\alpha, \beta$-unsaturated esters, such as 42 , do not undergo addition to aldehyde under the standard conditions (Fig 6d). The Rh-enolate derived from $\beta$-arylation of alkene 42 undergoes fast protonation to give $\mathbf{4 3}$ in $97 \%$ yield after 2.5 hours. In competition studies between alkene and diene, products from diene difunctionalization (44) are formed at a faster rate than products from alkene arylation (43) (Fig 6e). In the absence of aldehyde, 
diene $\delta$-arylation product is formed at similar rates to alkene $\beta$-arylation (Fig 6f). This is despite large differences in rates for independent experiments, where near quantitative alkene $\beta$-arylation occurs in less than 30 minutes. The slowing of alkene $\beta$-arylation rates in the presence of diene can be rationalized by the diene substrate preferentially binding to the Rh catalyst, effectively poisoning the $\beta$-arylation pathway.

Geometrical isomers of the standard diene $E, E-1$ are less productive substrates in $\alpha, \delta$ difunctionalization (Fig 6g). Z,E-1 slowly generates the same product stereoisomer as E,E-1 with reduced diastereoselectivity, while $E, Z$ - and Z,Z-1 are resistant to $\delta$-arylation. Under the reaction conditions $Z, E-1$ is converted to $E, E-1$ in a process catalyzed by $\mathrm{Rh}$ (see the SI for details). Improved yields when using $Z, E-1$ could be achieved by modifying the conditions to increase isomerization (2.5 $\mathrm{mol} \% \mathrm{Rh}$ at room temperature) and by using the more slowly reacting pinacol ester in place of the boronic acid as the nucleophile. Under the modified conditions, $66 \%$ yield of 2 was obtained in $97 \%$ ee and 82:18 $d r$ from $Z, E-1$, showing that crude mixture of diene products obtained by carbonyl olefination or cross-coupling, commonly obtained in 80:20 E,E/Z,E mixtures, can be using without removal of the $Z, E$ isomer.

Taken together, these mechanistic studies show that (Ph-tfb)Rh-allyl species are well suited to engage non proton electrophiles in multi-component coupling reactions triggered by $\delta$-arylation. By tailoring the reaction conditions (i.e. relatively high concentration and slight excess of aldehyde), diastereoselective coupling to aldehyde outpaces Rh-allyl $\eta^{1}-\eta^{3}-\eta^{1}$ isomerization, ultimately leading to the formation of one stereoisomer from the 16 possible outcomes. The reactivity of Rh-allyl species contrasts that of Rh-enolate intermediates for which electrophile trapping is stymied by rapid protonolysis. 


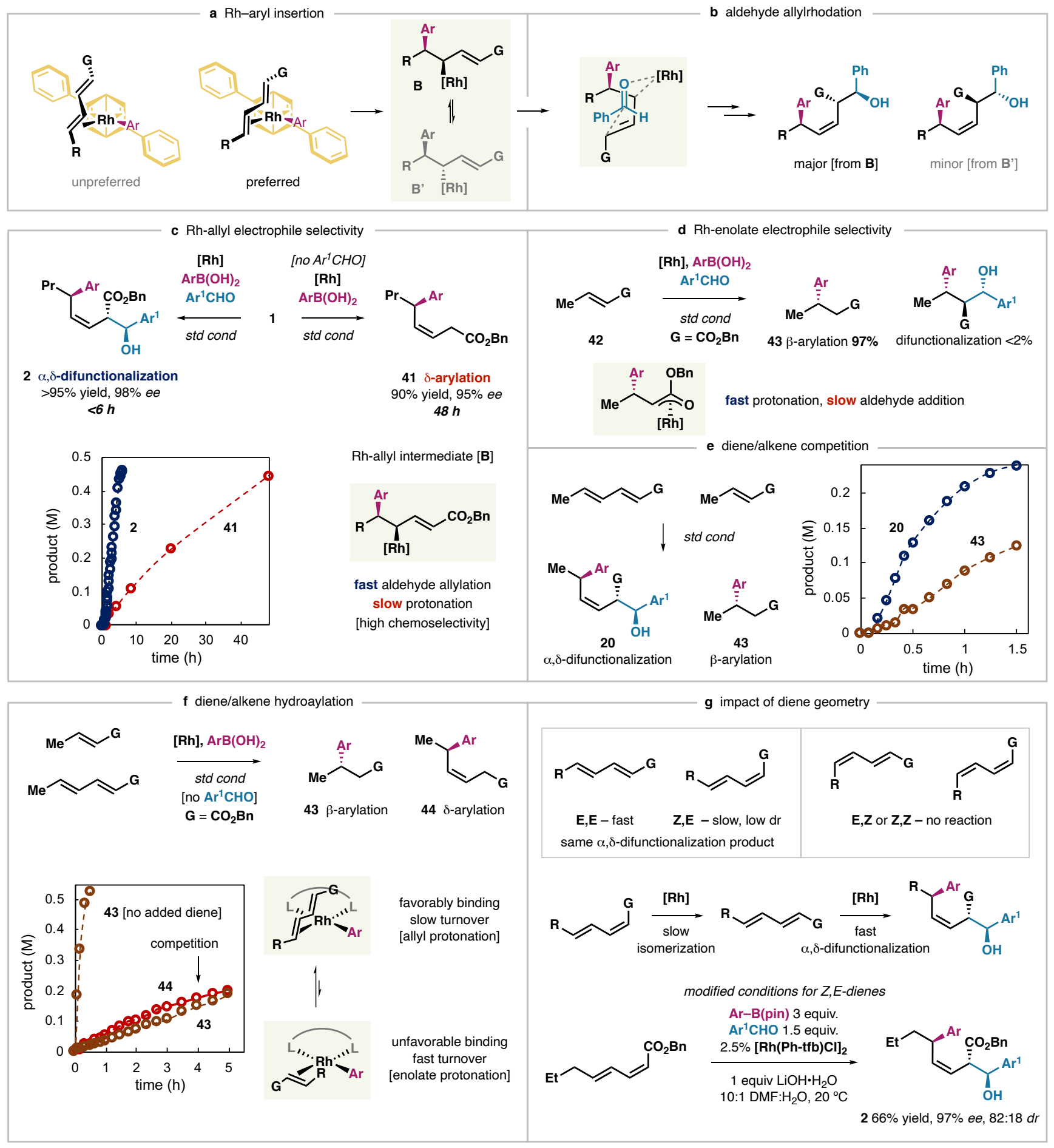

Figure 6. Mechanistic studies of the Rh-catalyzed $\alpha, \delta$-difunctionalization of dienes. (a) Stereochemical model for $\delta$-arylation. (b) Stereochemical model for aldehyde allylrhodation. (c) Reactivity comparison of aldehyde trapping vs protonation. (d) Reaction of electron-deficient alkenes under the standard conditions. (e) Diene/alkene competition studies in the presence of aldehyde (0.5 M total acceptor). (f) Diene/alkene hydroarylation rate and competition study. (f) Impact of dienoate geometry on reaction and Z,E-diene isomerization. 


\section{CONCLUSIONS}

Metal-catalyzed conjugate arylations are a practical and dependable reaction platform to generate stereocenters at positions remote from carbonyl or arene activating groups. This work shows that $\mathrm{Rh}$ catalysts featuring chiral benzobarrelene ligands can promote the enantioselective $\delta$-arylation of several classes of electron-poor dienes to generate Rh-allyl intermediates that can be coupled to aldehydes with high chemo- and stereoselectivity. The products contain a stereotriad separated by a Zalkene unit which would be tedious to prepare using a stepwise approach. The more general understanding of the reactivity of Rh-allyl intermediates formed by conjugate arylation should open this approach to alternative classes of reaction partners.

\section{ACKNOWLEDGEMENTS}

We thank NSERC Canada (RGPIN-2019-06050 and RGPAS-2019-00051 to R.J.L., PGS-D to C.J.C.C., USRA to S.G.), MITACS (Globalink Internship to B.S.), the University of Alberta, and the Province of Alberta (AGES fellowship to C.J.C.C.) for support. We thank Prof. Takahiro Nishimura (Osaka) for the gift of $[\mathrm{Rh}(\mathrm{Ph}-\mathrm{tfb}) \mathrm{Cl}]_{2}$ to initiate this project. Dr. Michael Ferguson (Alberta) is thanked for X-ray crystal structure determinations.

\section{REFERENCES}

1. (a) Jerphagnon, T.; Pizzuti, M. G.; Minnaard, A. J.; Feringa, B. L., Recent advances in enantioselective copper-catalyzed 1,4-addition. Chem. Soc. Rev. 2009, 38 (4), 1039-1075; (b) Berthon-Gelloz, G.; Hayashi, T., Rhodium- and Palladium-Catalyzed Asymmetric Conjugate Additions of Organoboronic Acids. In Boronic Acids, 2011; pp 263-313; (c) Alexakis, A.; Krause, N.; Woodward, S., Copper-Catalyzed Asymmetric Conjugate Addition. In Copper-Catalyzed Asymmetric Synthesis, 2014; pp 33-68; (d) Burns, A. R.; Lam, H. W.; Roy, I. D., Enantioselective, Rhodium-Catalyzed 1,4Addition of Organoboron Reagents to Electron-Deficient Alkenes. In Organic Reactions, 2017; pp 1415; (e) Zheng, K.; Liu, X.; Feng, X., Recent Advances in Metal-Catalyzed Asymmetric 1,4-Conjugate Addition (ACA) of Nonorganometallic Nucleophiles. Chem. Rev. 2018, 118 (16), 7586-7656.

2. (a) Hui, C.; Pu, F.; Xu, J., Metal-Catalyzed Asymmetric Michael Addition in Natural Product Synthesis. Chem. Eur. J. 2017, 23 (17), 4023-4036; (b) Vargová, D.; Némethová, I.; Šebesta, R., Asymmetric copper-catalyzed conjugate additions of organometallic reagents in the syntheses of natural compounds and pharmaceuticals. Org. Biomol. Chem. 2020, 18 (20), 3780-3796; (c) Edwards, H. J.; Hargrave, J. D.; Penrose, S. D.; Frost, C. G., Synthetic applications of rhodium catalysed 
conjugate addition. Chem. Soc. Rev. 2010, 39 (6), 2093-2105; (d) Jian, J.-H.; Zeng, H.-W.; Kuo, T.-S.; Wu, P.-Y.; Wu, H.-L., Asymmetric Synthesis of Functionalized Phenylalanine Derivatives via RhCatalyzed Conjugate Addition and Enantioselective Protonation Cascade. Org. Lett. 2019, 21 (23), 9468-9472; (e) Edelstein, E. K.; Rankic, D. A.; Dudley, C. C.; McMinn, S. E.; Adpressa, D. A., Synthesis of Proline Analogues via Rh-Catalyzed Asymmetric Conjugate Addition. ACS Catal. 2021, 11 (2), 743-749; (f) Simmons, E. M.; Mudryk, B.; Lee, A. G.; Qiu, Y.; Razler, T. M.; Hsiao, Y., Development of a Kilogram-Scale Process for the Enantioselective Synthesis of 3-Isopropenylcyclohexan-1-one via Rh/DTBM-SEGPHOS-Catalyzed Asymmetric Hayashi Addition Enabled by 1,3Diol Additives. Org. Process Res. Dev. 2017, 21 (10), 1659-1667; (g) Howell, G. P., Asymmetric and Diastereoselective Conjugate Addition Reactions: C-C Bond Formation at Large Scale. Org. Process Res. Dev. 2012, 16 (7), 1258-1272.

3. Csákÿ, A. G.; Herrán, G. d. I.; Murcia, M. C., Conjugate addition reactions of carbon nucleophiles to electron-deficient dienes. Chem. Soc. Rev. 2010, 39 (11), 4080-4102.

4. (a) den Hartog, T.; Harutyunyan, S. R.; Font, D.; Minnaard, A. J.; Feringa, B. L., Catalytic Enantioselective 1,6-Conjugate Addition of Grignard Reagents to Linear Dienoates. Angew. Chem. Int. Ed. 2008, 47 (2), 398-401; (b) Tissot, M.; Alexakis, A., Enantio- and Regioselective Conjugate Addition of Organometallic Reagents to Linear Polyconjugated Nitroolefins. Chem. Eur. J. 2013, 19 (34), 11352-11363; (c) Magrez-Chiquet, M.; Morin, M. S. T.; Wencel-Delord, J.; Drissi Amraoui, S.; Baslé, O.; Alexakis, A.; Crévisy, C.; Mauduit, M., Enantioselective 1,6-Conjugate Addition of Dialkylzinc Reagents to Acyclic Dienones Catalyzed by Cu-DiPPAM Complex-Extension to Asymmetric Sequential 1,6/1,4-Conjugate Addition. Chem. Eur. J. 2013, 19 (41), 13663-13667; (d) Guo, Y.; Kootstra, J.; Harutyunyan, S. R., Catalytic Regio- and Enantioselective Alkylation of Conjugated Dienyl Amides. Angew. Chem. Int. Ed. 2018, 57 (41), 13547-13550.

5. (a) Huang, Y.; Torker, S.; Li, X.; del Pozo, J.; Hoveyda, A. H., Racemic Vinylallenes in Catalytic Enantioselective Multicomponent Processes: Rapid Generation of Complexity through 1,6-Conjugate Additions. Angew. Chem. Int. Ed. 2019, 58 (9), 2685-2691; (b) Meng, F.; Li, X.; Torker, S.; Shi, Y.; Shen, X.; Hoveyda, A. H., Catalytic enantioselective 1,6-conjugate additions of propargyl and allyl groups. Nature 2016, 537 (7620), 387-393; (c) Shi, C.-Y.; Pan, Z.-Z.; Tian, P.; Yin, L., Copper(I)catalyzed asymmetric 1,6-conjugate allylation. Nat. Commun. 2020, 11 (1), 5480.

6. Sawano, T.; Ashouri, A.; Nishimura, T.; Hayashi, T., Cobalt-Catalyzed Asymmetric 1,6-Addition

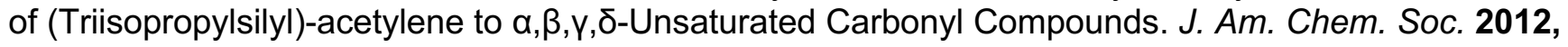
134 (46), 18936-18939.

7. (a) Nishimura, T.; Yasuhara, Y.; Sawano, T.; Hayashi, T., Iridium/Chiral Diene-Catalyzed

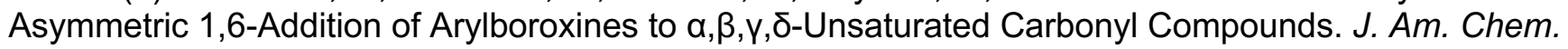
Soc. 2010, 132 (23), 7872-7873; (b) Nishimura, T.; Noishiki, A.; Hayashi, T., Electronic tuning of chiral diene ligands in iridium-catalyzed asymmetric 1,6 -addition of arylboroxines to $\delta$-aryl- $\alpha, \beta, \gamma, \delta$ unsaturated ketones. Chem. Commun. 2012, 48 (7), 973-975.

8. (a) Pierrot, D.; Marek, I., Synthesis of Enantioenriched Vicinal Tertiary and Quaternary Carbon Stereogenic Centers within an Acyclic Chain. Angew. Chem. Int. Ed. 2020, 59 (1), 36-49; (b) Eppe, G.; Didier, D.; Marek, I., Stereocontrolled Formation of Several Carbon-Carbon Bonds in Acyclic Systems. Chem. Rev. 2015, 115 (17), 9175-9206.

9. Vargová, D.; Némethová, I.; Plevová, K.; Šebesta, R., Asymmetric Transition-Metal Catalysis in the Formation and Functionalization of Metal Enolates. ACS. Catal. 2019, 9 (4), 3104-3143. 
10. (a) Pellissier, H., Enantioselective Copper-Catalyzed Domino Reactions. In Asymmetric Metal Catalysis in Enantioselective Domino Reactions, 2019; pp 1-55; (b) Cauble, D. F.; Gipson, J. D.; Krische, M. J., Diastereo- and Enantioselective Catalytic Carbometallative Aldol Cycloreduction: Tandem Conjugate Addition-Aldol Cyclization. J. Am. Chem. Soc. 2003, 125 (5), 1110-1111; (c) Guo, S.; Xie, Y.; Hu, X.; Huang, H., Highly Diastereo- and Enantioselective Tandem Reaction toward Functionalized Pyrrolidines with Multiple Stereocenters. Org. Lett. 2011, 13 (20), 5596-5599; (d) Guo, S.; Xie, Y.; Hu, X.; Xia, C.; Huang, H., Diastereo- and Enantioselective Catalytic Tandem Michael Addition/Mannich Reaction: Access to Chiral Isoindolinones and Azetidines with Multiple Stereocenters. Angew. Chem. Int. Ed. 2010, 49 (15), 2728-2731.

11. Nicolaou, K. C.; Tang, W.; Dagneau, P.; Faraoni, R., A Catalytic Asymmetric ThreeComponent 1,4-Addition/Aldol Reaction: Enantioselective Synthesis of the Spirocyclic System of Vannusal A. Angew. Chem. Int. Ed. 2005, 44 (25), 3874-3879.

12. Yoshida, K.; Ogasawara, M.; Hayashi, T., A New Type of Catalytic Tandem 1,4-Addition-Aldol Reaction Which Proceeds through an (Oxa-m-allyl)rhodium Intermediate. J. Am. Chem. Soc. 2002, 124 (37), 10984-10985.

13. Kina, A.; Iwamura, H.; Hayashi, T., A Kinetic Study on Rh/Binap-Catalyzed 1,4-Addition of Phenylboronic Acid to Enones: Negative Nonlinear Effect Caused by Predominant Homochiral Dimer Contribution. J. Am. Chem. Soc. 2006, 128 (12), 3904-3905.

14. For examples of enantioselective diene carbodifunctionalizations leading to the generation of one or two stereocenters see: (a) Wu, X.; Lin, H.-C.; Li, M.-L.; Li, L.-L.; Han, Z.-Y.; Gong, L.-Z., Enantioselective 1,2-Difunctionalization of Dienes Enabled by Chiral Palladium Complex-Catalyzed Cascade Arylation/Allylic Alkylation Reaction. J. Am. Chem. Soc. 2015, 137 (42), 13476-13479; (b) Li, G.; Huo, X.; Jiang, X.; Zhang, W., Asymmetric synthesis of allylic compounds via hydrofunctionalisation and difunctionalisation of dienes, allenes, and alkynes. Chem. Soc. Rev. 2020, 49 (7), 2060-2118; (c) Tao, Z.-L.; Adili, A.; Shen, H.-C.; Han, Z.-Y.; Gong, L.-Z., Catalytic Enantioselective Assembly of Homoallylic Alcohols from Dienes, Aryldiazonium Salts, and Aldehydes. Angew. Chem. Int. Ed. 2016, 55 (13), 4322-4326; (d) Xiong, Y.; Zhang, G., Enantioselective 1,2Difunctionalization of 1,3-Butadiene by Sequential Alkylation and Carbonyl Allylation. J. Am. Chem. Soc. 2018, 140 (8), 2735-2738; (e) Sato, Y.; Hinata, Y.; Seki, R.; Oonishi, Y.; Saito, N., NickelCatalyzed Enantio- and Diastereoselective Three-Component Coupling of 1,3-Dienes, Aldehydes, and Silanes Using Chiral N-Heterocyclic Carbenes as Ligands. Org. Lett. 2007, 9 (26), 5597-5599; (f) Okada, S.; Arayama, K.; Murayama, R.; Ishizuka, T.; Hara, K.; Hirone, N.; Hata, T.; Urabe, H., IronCatalyst-Switched Selective Conjugate Addition of Grignard Reagents: $\alpha, \beta, \gamma, \delta$-Unsaturated Amides as Versatile Templates for Asymmetric Three-Component Coupling Processes. Angew. Chem. Int. Ed. 2008, 47 (36), 6860-6864; (g) Lu, F.-D.; Lu, L.-Q.; He, G.-F.; Bai, J.-C.; Xiao, W.-J., Enantioselective Radical Carbocyanation of 1,3-Dienes via Photocatalytic Generation of Allylcopper Complexes. J. Am. Chem. Soc. 2021, 143 (11), 4168-4173; (h) Chai, W.; Zhou, Q.; Ai, W.; Zheng, Y.; Qin, T.; Xu, X.; Zi, W., Lewis-Acid-Promoted Ligand-Controlled Regiodivergent Cycloaddition of Pd-Oxyallyl with 1,3Dienes: Reaction Development and Origins of Selectivities. J. Am. Chem. Soc. 2021, 143 (9), 35953603; (i) Chen, J.; Miliordos, E.; Chen, M., Highly Diastereo- and Enantioselective Synthesis of 3,6'Bisboryl-anti-1,2-oxaborinan-3-enes: An Entry to Enantioenriched Homoallylic Alcohols with A Stereodefined Trisubstituted Alkene. Angew. Chem. Int. Ed. 2021, 60 (2), 840-848; For a diene difunctionalization that generates three stereocenters see: (j) Saito, N.; Kobayashi, A.; Sato, Y., Nickel-Catalyzed Enantio- and Diastereoselective Three-Component Coupling of 1,3-Dienes, Aldehydes, and a Silylborane Leading to a-Chiral Allylsilanes. Angew. Chem. Int. Ed. 2012, 51 (5), 1228-1231. 
15. Cooze, C.; Dada, R.; Lundgren, R. J., Direct Formic Acid Mediated Z-Selective Reductive Coupling of Dienes and Aldehydes. Angew. Chem. Int. Ed. 2019, 58 (35), 12246-12251.

16. For enantioselective diene functionalizations that lead to Z-alkene products see: (a) Lin, H.-C.; Xie, P.-P.; Dai, Z.-Y.; Zhang, S.-Q.; Wang, P.-S.; Chen, Y.-G.; Wang, T.-C.; Hong, X.; Gong, L.-Z., Nucleophile-Dependent Z/E- and Regioselectivity in the Palladium-Catalyzed Asymmetric Allylic C-H Alkylation of 1,4-Dienes. J. Am. Chem. Soc. 2019, 141 (14), 5824-5834; (b) Behlen, M. J.; Uyeda, C., C2-Symmetric Dinickel Catalysts for Enantioselective [4+1]-Cycloadditions. J. Am. Chem. Soc. 2020, 142 (41), 17294-17300; (c) Zhong, F.; Pan, Z.-Z.; Zhou, S.-W.; Zhang, H.-J.; Yin, L., Copper(I)Catalyzed Regioselective Asymmetric Addition of 1,4-Pentadiene to Ketones. J. Am. Chem. Soc. 2021, 143 (12), 4556-4562; (d) Gui, Y.-Y.; Hu, N.; Chen, X.-W.; Liao, L. L.; Ju, T.; Ye, J.-H.; Zhang, Z.; Li, J.; Yu, D.-G., Highly Regio- and Enantioselective Copper-Catalyzed Reductive Hydroxymethylation of Styrenes and 1,3-Dienes with CO2. J. Am. Chem. Soc. 2017, 139 (47), 17011-17014.

17. Nishimura, T.; Kumamoto, H.; Nagaosa, M.; Hayashi, T., The concise synthesis of chiral tfb ligands and their application to the rhodium-catalyzed asymmetric arylation of aldehydes. Chem. Commun. 2009, (38), 5713-5715.

18. (a) de la Herrán, G.; Murcia, C.; Csákÿ, A. G., Rhodium-Catalyzed Reaction of Aryl- and Alkenylboronic Acids with 2,4-Dienoate Esters: Conjugate Addition and Heck Reaction Products. Org. Lett. 2005, 7 (25), 5629-5632; (b) Moku, B.; Fang, W.-Y.; Leng, J.; Kantchev, E. A. B.; Qin, H.-L., $\mathrm{Rh}(\mathrm{I})$-Diene-Catalyzed Addition of (Hetero)aryl Functionality to 1,3-Dienylsulfonyl Fluorides Achieving Exclusive Regioselectivity and High Enantioselectivity: Generality and Mechanism. ACS Catal. 2019, 9 (11), 10477-10488.

19. (a) Groves, A.; Martínez, J. I.; Smith, J. J.; Lam, H. W., Remote Nucleophilic Allylation by Allylrhodium Chain Walking. Chem. Eur. J. 2018, 24 (51), 13432-13436; (b) Callingham, M.; Partridge, B. M.; Lewis, W.; Lam, H. W., Enantioselective Rhodium-Catalyzed Coupling of Arylboronic Acids, 1,3Enynes, and Imines by Alkenyl-to-Allyl 1,4-Rhodium(I) Migration. Angew. Chem. Int. Ed. 2017, 56 (51), 16352-16356; (c) Hepburn, H. B.; Lam, H. W., The Isomerization of Allylrhodium Intermediates in the Rhodium-Catalyzed Nucleophilic Allylation of Cyclic Imines. Angew. Chem. Int. Ed. 2014, 53 (43), 11605-11610.

20. For overviews of diolefin ancillary ligands see: (a) Esteruelas, M. A.; Oro, L. A., Iridium and rhodium complexes with tetrafluorobenzobarrelene diolefins. Coord. Chem. Rev. 1999, 193-195, 557618; (b) Nishimura, T.; Yasuhara, Y.; Nagaosa, M.; Hayashi, T., C2-Symmetric tetrafluorobenzobarrelenes as highly efficient ligands for the iridium-catalyzed asymmetric annulation of 1,3-dienes with 2-formylphenylboron reagents. Tetrahedron Asym. 2008, 19 (15), 1778-1783; (c) Defieber, C.; Grützmacher, H.; Carreira, E. M., Chiral Olefins as Steering Ligands in Asymmetric Catalysis. Angew. Chem. Int. Ed. 2008, 47 (24), 4482-4502; (d) Nagamoto, M.; Nishimura, T., Asymmetric Transformations under Iridium/Chiral Diene Catalysis. ACS Catal. 2017, 7 (1), 833-847.

21. Martínez, J. I.; Smith, J. J.; Hepburn, H. B.; Lam, H. W., Chain Walking of Allylrhodium Species Towards Esters During Rhodium-Catalyzed Nucleophilic Allylations of Imines. Angew. Chem. Int. Ed. 2016, 55 (3), 1108-1112.

22. Burés, J., Variable Time Normalization Analysis: General Graphical Elucidation of Reaction Orders from Concentration Profiles. Angew. Chem. Int. Ed. 2016, 55 (52), 16084-16087. 